\title{
Measurement of cross sections and couplings of the Higgs Boson using the ATLAS detector *
}

\author{
S. Honda ${ }^{\mathrm{a}}$, on behalf of the ATLAS collaboration \\ ${ }^{a}$ Faculty of Pure and Applied Sciences, University of Tsukuba
}

\begin{abstract}
After the discovery of the Higgs boson, the measurement of its coupling properties are of particular importance. In this talk measurement of the cross sections and couplings of the Higgs boson in bosonic and fermionic decay channels with the ATLAS detector are presented.
\end{abstract}

Keywords: ATLAS, Higgs

\section{Introduction}

Following the discovery of the Higgs boson by the ATLAS [1] and CMS [2] experiments, a broad range of measurements of its properties have been performed using proton-proton collision data produced by the CERN Large Hadron Collider (LHC) at centre-of-mass energies of $\sqrt{s}=7$ and $8 \mathrm{TeV}$ in Run 1 [3]. At Run 2, with the upgrade of the LHC energy to $\sqrt{s}=13 \mathrm{TeV}$ and ATLAS data taking for two years corresponding to an integrated luminosity of $36.1 \mathrm{fb}^{-1}$, Higgs boson properties such as kinematics of production processes can be measured with a factor of two improved precision than achieved in Run 1. The increased center-of-mass energy results in 2.4 times larger cross sections and improves sensitivity to various interesting physics processes, such as the Higgs boson decaying to di-muons or $b \bar{b}$, and the Higgs boson production in association with a topantitop quark pair. Preliminary new measurement and search results for standard-model Higgs processes obtained with $36.1 \mathrm{fb}^{-1}$ of data are summarized in this paper.

\footnotetext{
*Talk given at 20th International Conference in Quantum Chromodynamics (QCD 17), 3 - 7 july 2017, Montpellier - FR

Email address: shunsuke.honda@cern.ch (S. Honda)
}

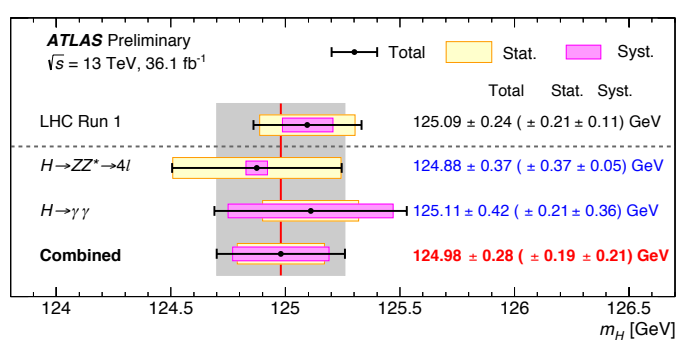

Figure 1: Summary of the Higgs boson mass measurements from the individual and combined analyses, compared to the combined Run 1 measurement by ATLAS and CMS [4].

\section{2. $\mathrm{H} \rightarrow \mathrm{ZZ}^{*}(\rightarrow 4 \ell)$ and $\gamma \gamma$}

Due to their clean signature and high $\mathrm{S} / \mathrm{B}$ ratio, $\mathrm{H} \rightarrow$ $\mathrm{ZZ}^{*}$ and $\gamma \gamma$ channels provide good signal sensitivities despite their small branching ratios. The largest background is non-resonant processes with continuous mass spectrum. Abilities to fully reconstruct the Higgs mass with excellent resolutions have been critical in establishing the Higgs signals.

These channels have been "re-discovered" in Run 2 data, and are used to measure various properties of the Higgs boson. The mass and couplings of the Higgs boson are measured by combining $\mathrm{H} \rightarrow \mathrm{ZZ}^{*}$ and $\gamma \gamma$ channels with $36.1 \mathrm{fb}^{-1}$ of data $[4,5]$. In the cross section 
measurement, results are published individually $[6,7]$.

\subsection{Mass Measurement}

The Higgs boson mass is measured to be $124.98 \pm$ $0.28 \mathrm{GeV}$ (Figure 1) [4]. The uncertainty is reduced by $32 \%$ from the ATLAS Run 1 measurement, $125.36 \pm$ $0.41 \mathrm{GeV}$ [8]. Main sources of systematic uncertainties on the combined result come from experimental effects such as LAr calibrations and energy loss estimate in materials in the $\mathrm{H} \rightarrow \gamma \gamma$ channel.

\subsection{Coupling Measurement}

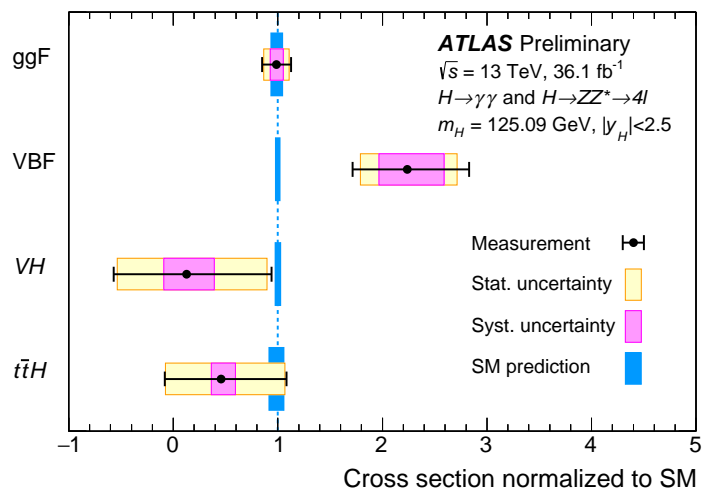

Figure 2: Cross sections for ggF, VBF, VH, and $\mathrm{t} \overline{\mathrm{t}} \mathrm{H}$ processes measured with assumption of predicted branching ratios [5], normalized to the standard model predictions.

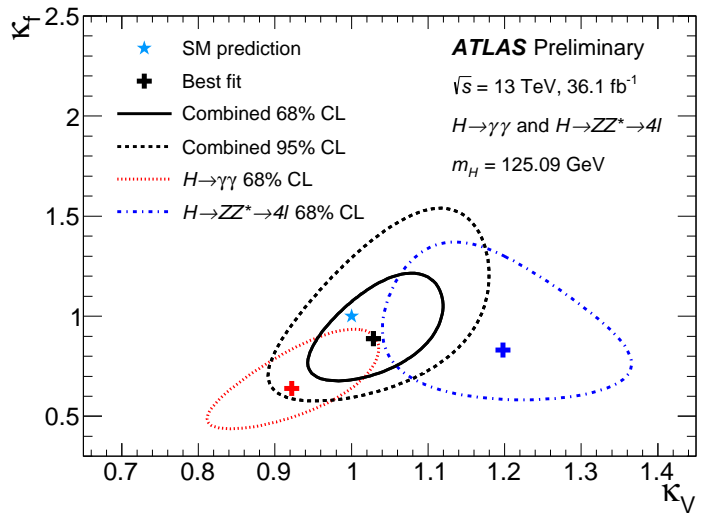

Figure 3: Contours at $68 \%$ and $95 \%$ confidence levels in the plane of Higgs couplings to fermions $\left(\kappa_{\mathrm{f}}\right)$ and weak-bosons $\left(\kappa_{\mathrm{V}}\right)$ [5]

Combined measurements of Higgs boson production cross sections and branching ratios are presented in [5]. The results are shown in Figure 2 for the individual production processes of gluon fusion ( $\mathrm{ggF})$, vector-boson fusion (VBF), VH, and $\bar{t} \bar{H}$. No significant deviations from the expectations are observed, although VBF is higher by about 2 standard deviations from the standard model hypothesis.

Coupling measurements are expressed in terms of $\kappa$, coupling strength relative to the prediction. Two models are considered in performing a two-parameter likelihood fit. The first model uses the coupling strength to the fermion, $\kappa_{\mathrm{f}}$, and to the weak-boson, $\kappa_{\mathrm{V}}$, as free parameters. The $\mathrm{H} \rightarrow \mathrm{ZZ}^{*}$ mode is proportional to $\kappa_{\mathrm{V}}{ }^{2}$, while the $\mathrm{H} \rightarrow \gamma \gamma$ mode depends on $\kappa_{\mathrm{V}}{ }^{2} \kappa_{\mathrm{f}}^{2}$. A small positive correlation is seen in the fit result (Figure 3) due in part to the destructive interference between the topquark and $\mathrm{W}$-boson loops in the $\mathrm{H} \rightarrow \gamma \gamma$ mode. The fit to data excludes negative $\kappa_{\mathrm{f}}$ value at a confidence level (CL) well beyond $95 \%$.

The second model uses the coupling strength to the photon, $\kappa_{\gamma}$, and to the gluon, $\kappa_{\mathrm{g}}$, as free parameters, and the result shows no evidence for deviations from the prediction.

\subsection{Cross Section Measurement}

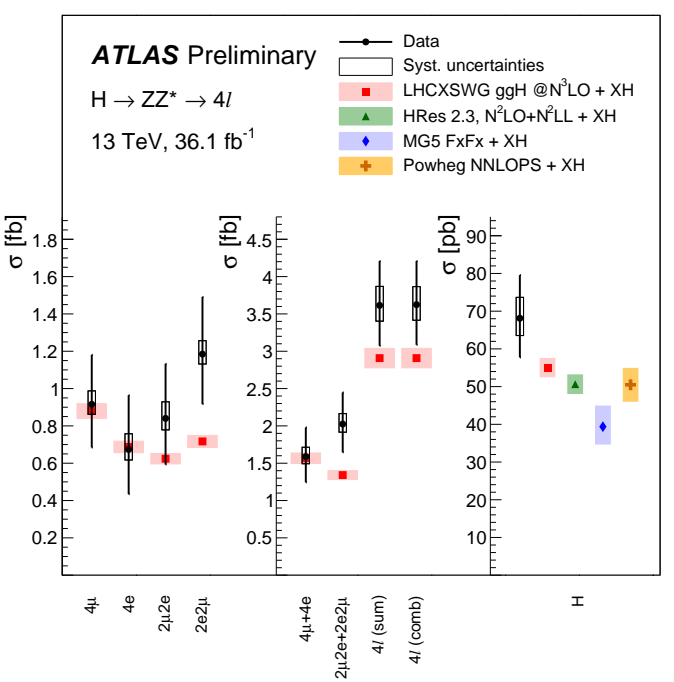

Figure 4: The fiducial cross sections (left two panels) and total cross section (right panel) of the Higgs boson production measured in the $4 \ell$ final state. The inclusive fiducial cross section is measured as the sum of all channels, as well as by combining the per-channel measurements assuming SM branching ratios [6].

Inclusive and differential fiducial cross sections of Higgs-boson production are measured in $\mathrm{H} \rightarrow$ $\mathrm{ZZ}^{*}$ and $\gamma \gamma$ channels $[6,7]$. All of them indicate good compatibility with the standard model predictions, although they have still large statistical uncertainties. 
Figure 4 shows inclusive cross sections in the $\mathrm{H} \rightarrow$ $\mathrm{ZZ}^{*}$ mode. Figure 5 shows the differential cross sections in terms of $\mathrm{p}_{\mathrm{T}, 4 \ell}$, which corresponds to $\mathrm{p}_{\mathrm{T}, \mathrm{Higgs}}$, and thus can probe the production kinematics of the Higgs boson. This is the most precise measurement for $\mathrm{p}_{\mathrm{T}, \mathrm{Higgs}}$. Differential distributions of the leading jet $\mathrm{p}_{\mathrm{T}}$ and number of jets are also measured in Figures 6 and 7, which can probe QCD activities.

The data tend to be larger than predictions in higher $\mathrm{p}_{\mathrm{T}}$ or jet-multiplicity bins in differential distributions. These deviations should be studied further with more accurate QCD calculations and more data to reduce statistical uncertainties. The same tendencies are seen also in the $\mathrm{H} \rightarrow \gamma \gamma[7]$.

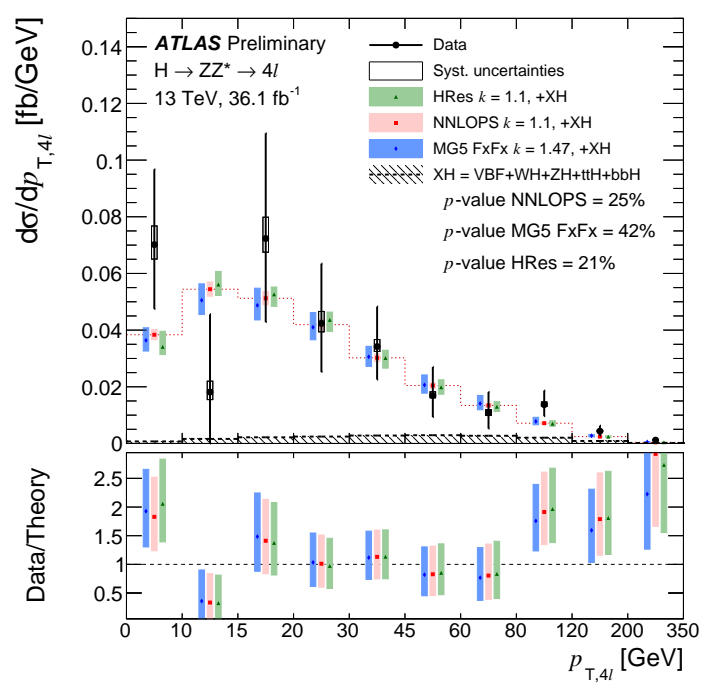

Figure 5: Differential fiducial cross sections for the transverse momentum of the Higgs boson [6].

\section{3. $\mathbf{H} \rightarrow \mu^{+} \mu^{-}$}

Although the $\mathrm{H} \rightarrow \mu^{+} \mu^{-}$mode is predicted to have a very small branching ratio, $0.022 \%$, the final state is clean due to the existence of two isolated muons. To maximize the sensitivity, eight orthogonal categories are defined by using BDT for two VBF categories, and different di-muon $\mathrm{p}_{\mathrm{T}}$ and muon $\eta$ ranges for six ggF categories. When Run 1 and Run 2 data are combined, observed (expected) upper limit on the signal strength parameter $\mu$ of the $\mathrm{H} \rightarrow \mu^{+} \mu^{-}$is 2.8 (2.9) at the $95 \%$ CL [9]. Since the statistical uncertainty is dominant, the full Run 2 dataset is not sufficient for an observation of this channel.

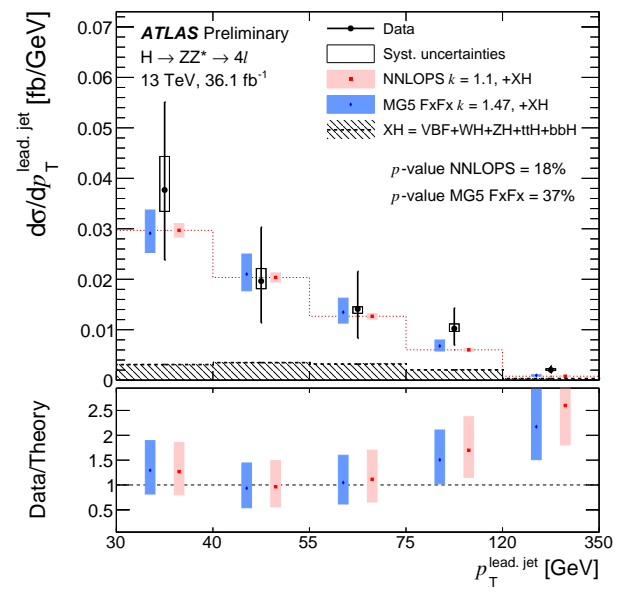

Figure 6: Differential fiducial cross sections for the transverse momentum of the leading jet [6].

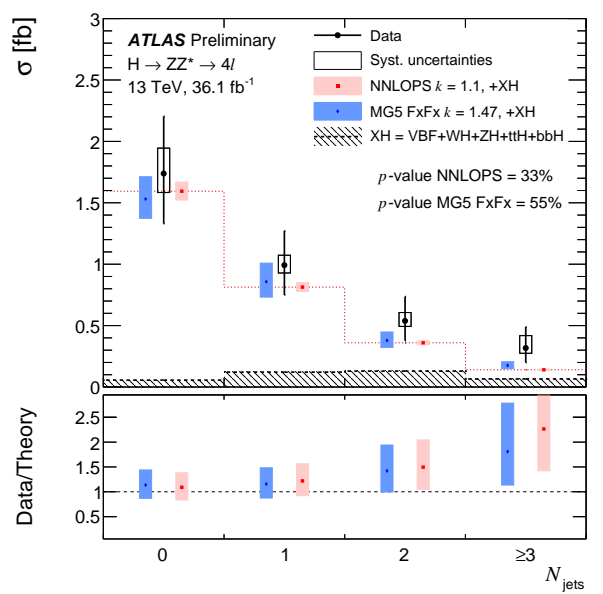

Figure 7: Differential fiducial cross sections for the number of jets [6].

\section{4. $\mathbf{H} \rightarrow \mathbf{b} \overline{\mathbf{b}}$}

The $\mathrm{H} \rightarrow \mathrm{b} \overline{\mathrm{b}}$ mode is predicted to have the highest branching ratio, 57.5\%. However, there are large background sources which are difficult to model well. Event selections and trigger decisions should be carefully designed to keep both $\mathrm{S} / \mathrm{B}$ ratio and signal efficiency high. A multivariate analysis using boosted decision tree (BDT) can maximize the separation of signal from large background. Simultaneous fit is generally adopted to measure the cross section of the Higgs boson with an un-modeled background processes.

\subsection{VH Process}

A search for $\mathrm{H} \rightarrow \mathrm{b} \bar{b}$ produced in association with a weak boson is performed [10]. Final states containing 
zero, one and two charged leptons (electrons or muons) are considered, targeting $\mathrm{Z} \rightarrow v v, \mathrm{~W} \rightarrow \ell v$ and $\mathrm{Z} \rightarrow \ell \ell$, respectively. Main background processes are $\mathrm{V}+\mathrm{HF}$ and $\mathrm{t} \overline{\mathrm{t}}$.

The definition of signal and control regions are given in Table 1. The signal regions are categorized with the

Table 1: Definition of signal and control regions and their distributions used in the simultaneous likelihood fit to obtain the cross section of $\mathrm{VH}(\mathrm{H} \rightarrow \mathrm{bb})[10]$

\begin{tabular}{|c|c|c|c|c|c|}
\hline \multirow{3}{*}{ Channel } & \multirow{3}{*}{$\mathrm{SR} / \mathrm{CR}$} & \multicolumn{4}{|c|}{ Categories } \\
\hline & & \multicolumn{2}{|c|}{$75 \mathrm{GeV}<p_{\mathrm{T}}^{V}<150 \mathrm{GeV}$} & \multicolumn{2}{|c|}{$p_{\mathrm{T}}^{V}>150 \mathrm{GeV}$} \\
\hline & & 2 jets & 3 jets & 2 jets & 3 jets \\
\hline$\overline{\text { 0-le }}$ & $\overline{\mathrm{SR}}$ & - & - & BDT & $\overline{\mathrm{BDT}}$ \\
\hline & $\mathrm{SP}$ & - & - & BDT & BDT \\
\hline 2-lepton & $\mathrm{SR}$ & BDT & BDT & BDT & $\mathrm{BDT}$ \\
\hline 1-lepton & $W+$ HF CR & - & - & Yield & Yield \\
\hline 2-lepton & $e \mu \mathrm{CR}$ & $m_{b b}$ & $m_{b b}$ & Yield & $m_{b b}$ \\
\hline
\end{tabular}

number of leptons, number of jets and transverse momentum of the weak boson $\left(\mathrm{p}_{\mathrm{T}}^{\mathrm{V}}\right)$, where $\mathrm{p}_{\mathrm{T}}^{\mathrm{V}}$ is defined in each region as :

- transverse momentum of the two charged lepton system in the 2-lepton channel.

- magnitude of the vectorial sum of the missing transverse energy and the charged lepton's transverse momentum in the 1-lepton channel.

- missing transverse energy in the 0-lepton channel.

The 2-lepton region with high $\mathrm{p}_{\mathrm{T}}^{\mathrm{V}}$ has the highest sensitivity. Its BDT distribution is shown in Figure 8.

The control region for enhanced $\mathrm{W}+\mathrm{HF}$ background is defined by requiring $\mathrm{m}_{\mathrm{b} \overline{\mathrm{b}}}<75 \mathrm{GeV}$ and $\mathrm{m}_{\mathrm{top}}>$ $225 \mathrm{GeV}$ in the 1-lepton channel, which is further subdivided into the 2- and 3-jet categories.

The control region, $\mathrm{e} \mu \mathrm{CR}$, is defined to control $\mathrm{t} \overline{\mathrm{t}}$ and $\mathrm{Wt}$ background by requiring different flavors and same charges in the 2-lepton channel. This region is subdivided to four regions by the number of jets and $\mathrm{p}_{\mathrm{T}}^{\mathrm{V}}$.

The results for the signal strength parameter $\mu$ are shown in Figure 9. When all channels are combined, the probability that observed result can be explained as background alone is $0.019 \%$. In the presence of a Higgs boson with the SM signal strength, the expected probability value is $0.12 \%$. The observation corresponds to an excess with a significance of 3.5 standard deviations, to be compared with an expectation of 3.0 standard deviations. With Run 1 and Run 2 combination, the observed signal strength parameter $\mu$ is $0.90_{-0.26}^{+0.28}$, corresponding to an observed (expected) significance of 3.6 (4.0) standard deviations.

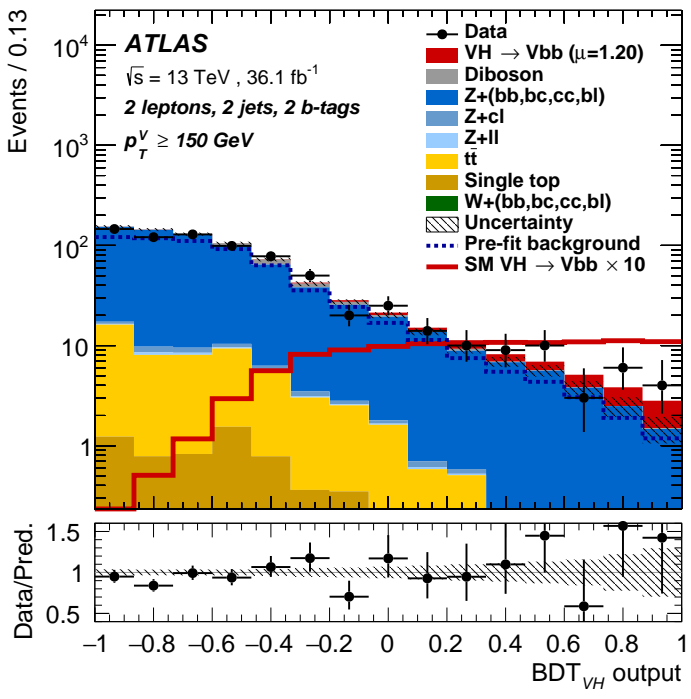

Figure 8: BDT output score in the highest sensitive region containing 2 leptons and required $\mathrm{pT}$ of the vector boson more than $150 \mathrm{GeV}[10]$.

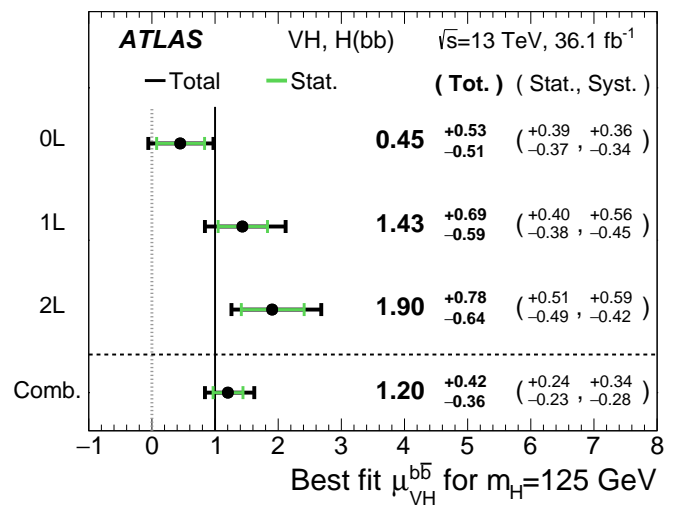

Figure 9: The fitted values of the Higgs boson signal strength parameter $\mu$ assuming Higgs mass of $125 \mathrm{GeV}$ for the 0-, 1- and 2-lepton channels and their combination. The signal strength parameter $\mu$ is defined by the ratio of the observed cross section of $\mathrm{VH}(\mathrm{H} \rightarrow \mathrm{bb})$ and the one predicted by the standard model. The compatibility of the individual signal strengths is $10 \%$ [10].

\section{2. $\mathrm{t} \overline{\mathrm{t}} \mathrm{H}$ Process}

A search for the $\mathrm{H} \rightarrow \mathrm{b} \overline{\mathrm{b}}$ produced in association with a top-antitop quark pair is also performed, although this analysis has been published with $13 \mathrm{fb}^{-1}$ of Run 2 data [11]. The $\bar{t}_{\mathrm{t}} \mathrm{H}$ with $\mathrm{H} \rightarrow \mathrm{bb}$ analysis has nine (five) regions divided by the number of jets and b-jets in 1(2-) lepton channels with minimum selections intended not to kill signal events. Since main background sources are $\mathrm{t} \overline{\mathrm{t}}+\geq 1 \mathrm{~b}$ and $\mathrm{t} \overline{\mathrm{t}}+\geq 1 \mathrm{c}$ processes, which are not modelled well in the MC simulation, their normaliza- 
tion correction factors are treated as free parameters in the fit along with the cross section of the $t \bar{t} \mathrm{H}(\mathrm{H} \rightarrow \mathrm{bb})$ process. The kinematics of the $\bar{t} \bar{t}+b \bar{b}$ process is very similar to the signal and makes it difficult to separate. Therefore, the BDT is adopted in five regions with high $\mathrm{S} / \mathrm{B}$ ratio to maximize their significances. The most sensitive region is $\geq 6$ jets and $\geq 4$ b-jets $(\geq 6 j, \geq 4 b)$ in 1-lepton channel, of which BDT output distribution is shown in Figure 10.

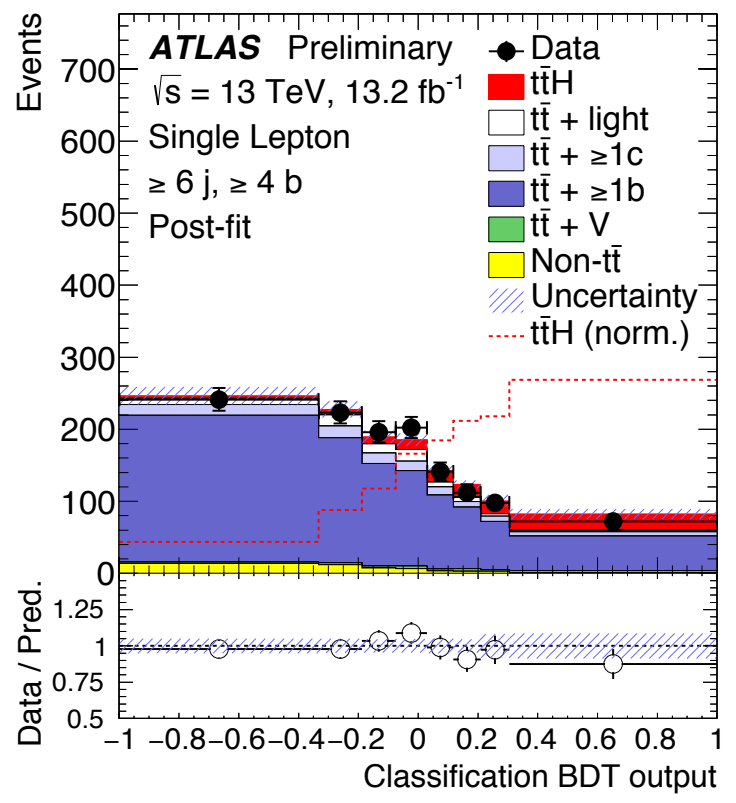

Figure 10: Comparison of data and prediction for the BDT discriminant variable in $\geq 6 \mathrm{j}, \geq 4 \mathrm{~b}$ in 1 -lepton channel. This region has the highest sensitivity in this analysis [11].

The observed Higgs boson signal strength parameter $\mu$ is $2.1_{-0.9}^{+1.0}$. The statistical uncertainty $\left({ }_{-0.5}^{+0.5}\right)$ is significantly smaller than the systematic uncertainties. To improve this analysis further with $36.1 \mathrm{fb}^{-1}$, better control of the modeling of $\mathrm{t} \overline{\mathrm{t}}+\geq 1 \mathrm{~b}$ and $\mathrm{t} \overline{\mathrm{t}}+\geq 1 \mathrm{c}$, as well as optimization of region definitions, are required to increase the purity of the signal.

\section{5. t'̄tH Production}

In the previous section, the Higgs boson production associated with a top-antitop quark pair is described using only the $\mathrm{H} \rightarrow \mathrm{b} \bar{b}$ mode. In this section, all three channels, $\mathrm{H} \rightarrow \gamma \gamma, \mathrm{H} \rightarrow\left(\mathrm{WW}, \mathrm{ZZ}^{*}, \tau \tau\right)$, and $\mathrm{H} \rightarrow \mathrm{b} \overline{\mathrm{b}}$, are used for the search for $\mathrm{t} \overline{\mathrm{t}} \mathrm{H}$ production, which is also published with $13 \mathrm{fb}^{-1}$ data [12].

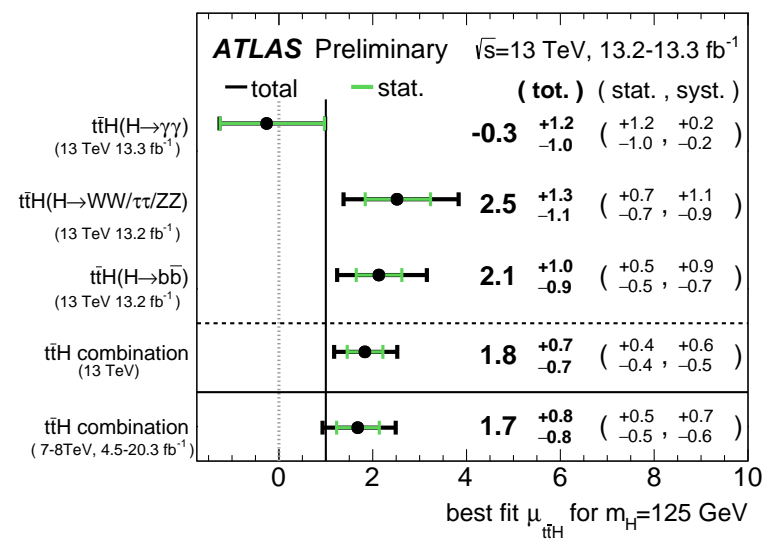

Figure 11: Summary of the observed $t \bar{t} \mathrm{H}$ signal strength measurements from the individual analyses and for their combination assuming mass of the Higgs boson at $125 \mathrm{GeV}$. The total (tot.), statistical (stat.) and systematic (syst.) uncertainties are shown. The observed signal strength from the Run 1 is also shown at the last column [12].

The result is summarized in Figure 11. The observed significance is 2.8 standard deviations away from the background only hypothesis, while the expected value is 1.8 .

Only the $\overline{\mathrm{t}} \mathrm{H}(\mathrm{H} \rightarrow \gamma \gamma)$ channel has been published with $36.1 \mathrm{fb}^{-1}$ as combined with $t H$ processes, which is shown in Figure 12 [7]. The observed signal strength parameter $\mu$ is $0.5_{-0.6}^{+0.6}$. The uncertainty is reduced by a factor of two from the previous result. $\mathrm{H} \rightarrow\left(\mathrm{WW}, \mathrm{ZZ}^{*}, \tau \tau\right)$ and $\mathrm{b} \overline{\mathrm{b}}$ modes will be also published with various improvements for $36.1 \mathrm{fb}^{-1}$. These improvements will allow a direct measurement of the Yukawa coupling of the top quark with a good precision, possibly limiting the beyond standard model parameters.

\section{Conclusions}

Various Higgs property measurements are being performed by the ATLAS experiment at $\sqrt{\mathrm{s}}=$ 7, 8 and $13 \mathrm{TeV}$. The Run 2 data corresponding to an integrated luminosity of $36.1 \mathrm{fb}^{-1}$ allow a more precise determination of the Higgs properties. The observed Higgs properties are compared to up-to-date, highly accurate theory calculations for various production processes and decay channels. The observed couplings and differential distributions are consistent with the standard model predictions, although statistical uncertainties limit their precisions. Mass measurements are also updated with a $32 \%$ reduction in the uncertainty from Run 1 measurements. 


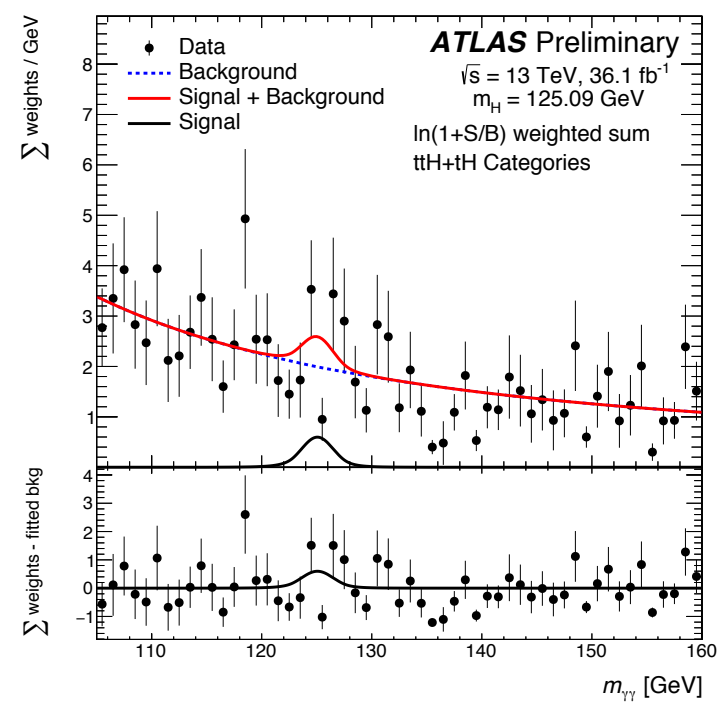

Figure 12: Diphoton invariant mass spectra passing $\mathrm{t} \overline{\mathrm{t}} \mathrm{H}$ and $\mathrm{tH}$ event selections. Each event is weighted by the $\ln (1+\mathrm{S} 90 / \mathrm{B} 90)$ ratio of the expected signal (S90) and background (B90) of the $90 \%$ signal quantile in the category it belongs to. The solid red curve shows the fitted signal-plus-background model, while the background(signal) component of the fit is shown with the dotted-blue(solid-black) curve [7].

The Yukawa couplings to the muon, the bottom quark, and the top quark are now intense areas of studies aiming at their observations or more stringent limits. An evidence for the bottom Yukawa coupling has been observed at $3.6 \sigma$. The $\bar{t} \bar{t} H$ process and other new results will be available in the near future, which allow more precise measurements of their couplings and other properties of the Higgs boson.

\section{References}

[1] ATLAS Collaboration, JINST 3 (2008) S08003.

[2] CMS Collaboration, JINST 3 (2008) S08004.

[3] ATLAS and CMS Collaborations, JHEP 1608 (2016) 045.

[4] ATLAS Collaboration, ATLAS-CONF-2017-046, https://cds.cern.ch/record/2273853/.

[5] ATLAS Collaboration, ATLAS-CONF-2017-047, https://cds.cern.ch/record/2273854/.

[6] ATLAS Collaboration, ATLAS-CONF-2017-032, https://cds.cern.ch/record/2265796/.

[7] ATLAS Collaboration, ATLAS-CONF-2017-045, https://cds.cern.ch/record/2273852/.

[8] ATLAS Collaboration, Phys. Rev. D 90 (2014) 052004.

[9] ATLAS Collaboration, Phys. Rev. Lett. 119 (2017) 051802.

[10] ATLAS Collaboration, arXiv:1708.03299 [hep-ex].

[11] ATLAS Collaboration, ATLAS-CONF-2016-080, https://cds.cern.ch/record/2206255/.

[12] ATLAS Collaboration, ATLAS-CONF-2016-068, https://cds.cern.ch/record/2206211/. 